\title{
Acute left main coronary artery thrombosis as an initial presentation of systemic lupus erythematosus
}

\author{
Kang Un Choi, Ung Kim \\ Division of Cardiology, Department of Internal Medicine, Yeungnam University College of Medicine, Daegu, Korea
}

Left main coronary artery (LMCA) thrombosis is rare and the cause should be determined. A previously healthy young man presented with severe chest pain and dyspnea. The electrocardiogram showed typical ST-segment elevation myocardial infarction with clinical instability. Emergency coronary angiography revealed complete LMCA occlusion by thrombosis. After reperfusion, the patient was admitted to the cardiac care unit. He was diagnosed with hemolytic anemia and tested positive for antinuclear antibodies. Systemic lupus erythematosus (SLE) and LMCA disease due to systemic thrombosis were diagnosed. Steroids were started and the patient was discharged without complications. We report this rare case of LMCA thrombosis as an initial presentation of SLE.

Keywords: Coronary thrombosis; Systemic lupus erythematosus

\section{INTRODUCTION}

Acute left main coronary artery thrombosis (LMCA) in young adults is rare and its etiology should be determined because the outcome can be catastrophic [1]. Systemic lupus erythematosus (SLE) is a chronic autoimmune disease with multisystem involvement. In most cases, initial presentations are non-cardiac and include arthralgias, arthritis, and/or skin involvement. However, an initial cardiac presentation can include tamponade [2] or myopericarditis [3].

The incidence of ischemic heart disease ranges from $8 \%$ to $16 \%$ in SLE patients but few reports have described STsegment elevation acute myocardial infarction (AMI) as an initial presentation [4-6. We report a case in which a relatively

Received: February 21, 2018, Revised: March 14, 2018

Accepted: March 28, 2018

Corresponding Author: Ung Kim, Division of Cardiology, Department of Internal Medicine, Yeungnam University College of Medicine, 170, Hyunchung-ro, Nam-gu, Daegu 42415, Korea

Tel: +82-53-620-3832, Fax: +82-53-654-8386

E-mail:woongwa@yu.ac.kr young (40-year-old) man developed acute LMCA thrombosis as an initial presentation of SLE.

\section{CASE}

A 40-year-old, previously healthy man was admitted to the emergency room with sudden onset chest pain and dyspnea on exertion. The patient denied history of smoking, hypertension, or diabetes mellitus. Initial examination showed blood pressure (BP) 72/50 $\mathrm{mmHg}$, pulse rate 112 beats/min, and respiratory rate 22 breaths/min. The initial electrocardiogram showed normal sinus rhythm with ST-segment elevation in leads I, aVL, and V1-3, and ST-segment depression in leads II, III, and aVF (Fig. 1). The troponin I ( $2.4 \mathrm{ng} / \mathrm{mL})$ and creatine kinase-MB $(9.8 \mathrm{ng} / \mathrm{mL})$ levels were elevated. Emergency coronary angiography showed complete left main coronary occlusion by a huge thrombus (Fig. 2A). Thrombus aspiration was performed several times (Figs. 2B, 2C), and thrombolysis in myocardial infarction grade 3 flow was achieved (Fig. 2D). Intravascular ultrasound was not performed because of unstable BP. The patient was transferred to the cardiac care

Copyright $($ C) 2018 Yeungnam University College of Medicine

This is an Open Access article distributed under the terms of the Creative Commons Attribution Non-Commercial License (http://creativecommons.org/licenses/by-nc/4.0/) which permits unrestricted non-commercial use, distribution, and reproduction in any medium, provided the original work is properly cited. 


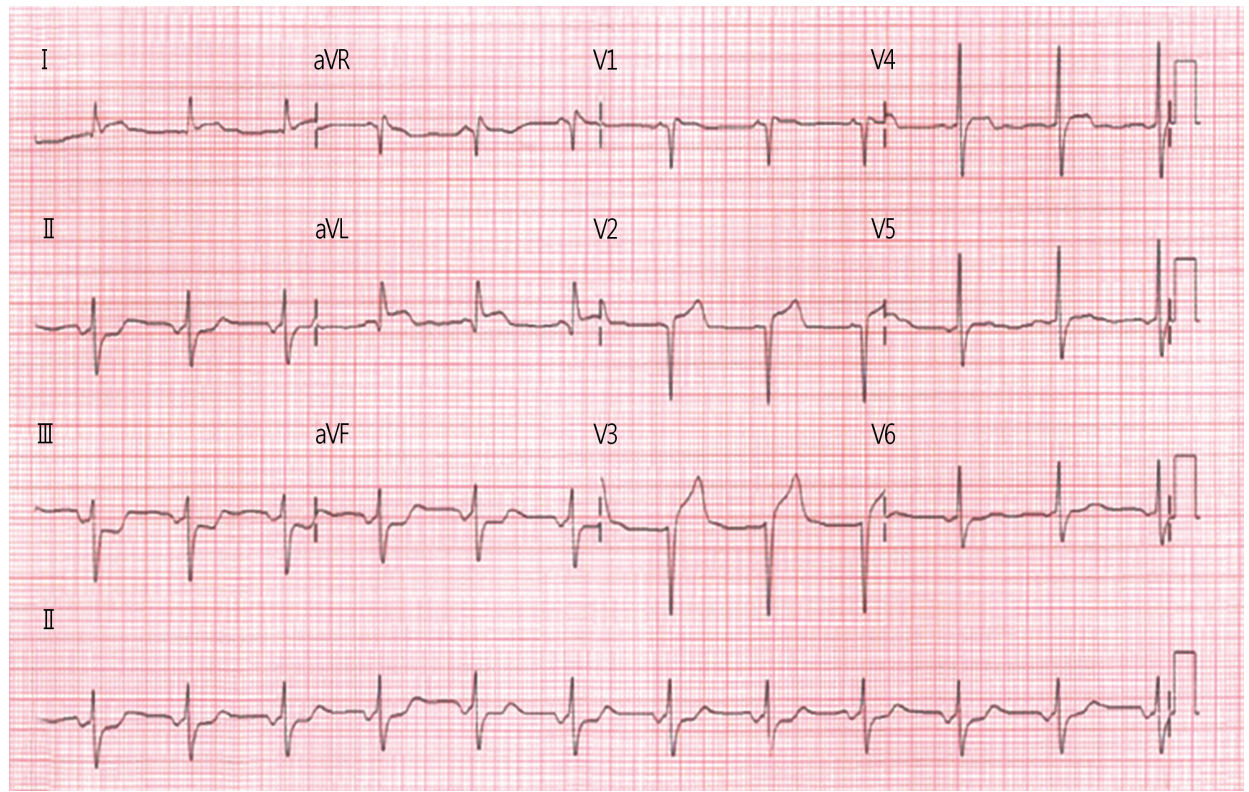

Fig. 1. Initial electrocardiogram findings. There is normal sinus rhythm with ST elevation in leads I, aVL and V1-3, and ST segment depression in leads II, III and aVF.

unit with inotropic support, and BP 110/80 $\mathrm{mmHg}$, pulse rate 72 beats/min, and respiratory rate 18 breaths/min. A simple chest radiograph showed pulmonary edema and a left pleural effusion. Although the white blood cell count, platelet count, and electrolytes were normal, the complete blood count revealed macrocytic anemia with hemoglobin $9.2 \mathrm{~g} / \mathrm{dL}$; blood urea nitrogen and creatinine were elevated at $38.4 \mathrm{mg} / \mathrm{dL}$ and $2.37 \mathrm{mg} / \mathrm{dL}$, respectively. An elevated antinuclear antibody titer (1:1,280, speckled) and decreased C4 (6.6 mg/dL), C3c (44.9 mg/dL), and haptoglobin (13 mg/dL) levels were noted. A peripheral blood smear showed evidence of hemolysis(po lychromasia). Transthoracic echocardiography showed anterior and lateral wall hypokinesis with an ejection fraction of $38 \%$. Five days after the index procedure, serum hemoglobin was decreased to $6.7 \mathrm{~g} / \mathrm{dL}$. The Coombs test was positive. The patient had serositis, renal involvement, hemolytic anemia, positive antineutrophil antibody, and low complement levels, consistent with a diagnosis of SLE. We immediately initiated prednisone $(1 \mathrm{~g} / \mathrm{kg} /$ day, orally). Five days after steroid pulse therapy, the patient was stabilized and laboratory tests improved with hemoglobin $10 \mathrm{~g} / \mathrm{dL}$ and haptoglobin $61 \mathrm{mg} / \mathrm{dL}$. Repeat coronary angiography was performed 11 days after admission for further evaluation and treatment of the LMCA. The previous aspiration sites in the proximal left anterior descending artery (LAD) and distal LMCA showed good pa- tency. Significant stenosis in the distal right coronary artery (RCA) was noted, and percutaneous coronary intervention with a drug-eluting stent $(4.0 \times 20 \mathrm{~mm}$, Promus Element, Boston Scientific, Natick, MA, USA) was performed. The patient was discharged with aspirin, clopidogrel, warfarin, statin, betablocker, angiotensin-converting enzyme inhibitor, and steroid (60 mg/day) prescriptions. During follow-up, warfarin was stopped after one month and dual antiplatelet therapy with aspirin and clopidogrel was continued for 1 year. Follow-up coronary angiography at 1 year after the index procedure found no significant stenosis or thrombus in the LMCA, proximal LAD, or stented RCA (Fig. 3). The patient has been followed up without any complications or recurrences.

\section{DISCUSSION}

Our case showed that acute LMCA thrombosis can present as the initial symptom of previously unrecognized SLE. The incidence of LMCA thrombosis is unknown, but is thought to be $0.8 \%$ or lower. This is because the first presentation may be sudden cardiac death. In this case, the patient arrived at the hospital within 30 minutes after the onset of symptoms and reperfusion was achieved within 90 minutes. Because the thrombus completely occluded the LMCA, delayed diagnosis could have been fatal. The incidence of AMI in an SLE cohort 

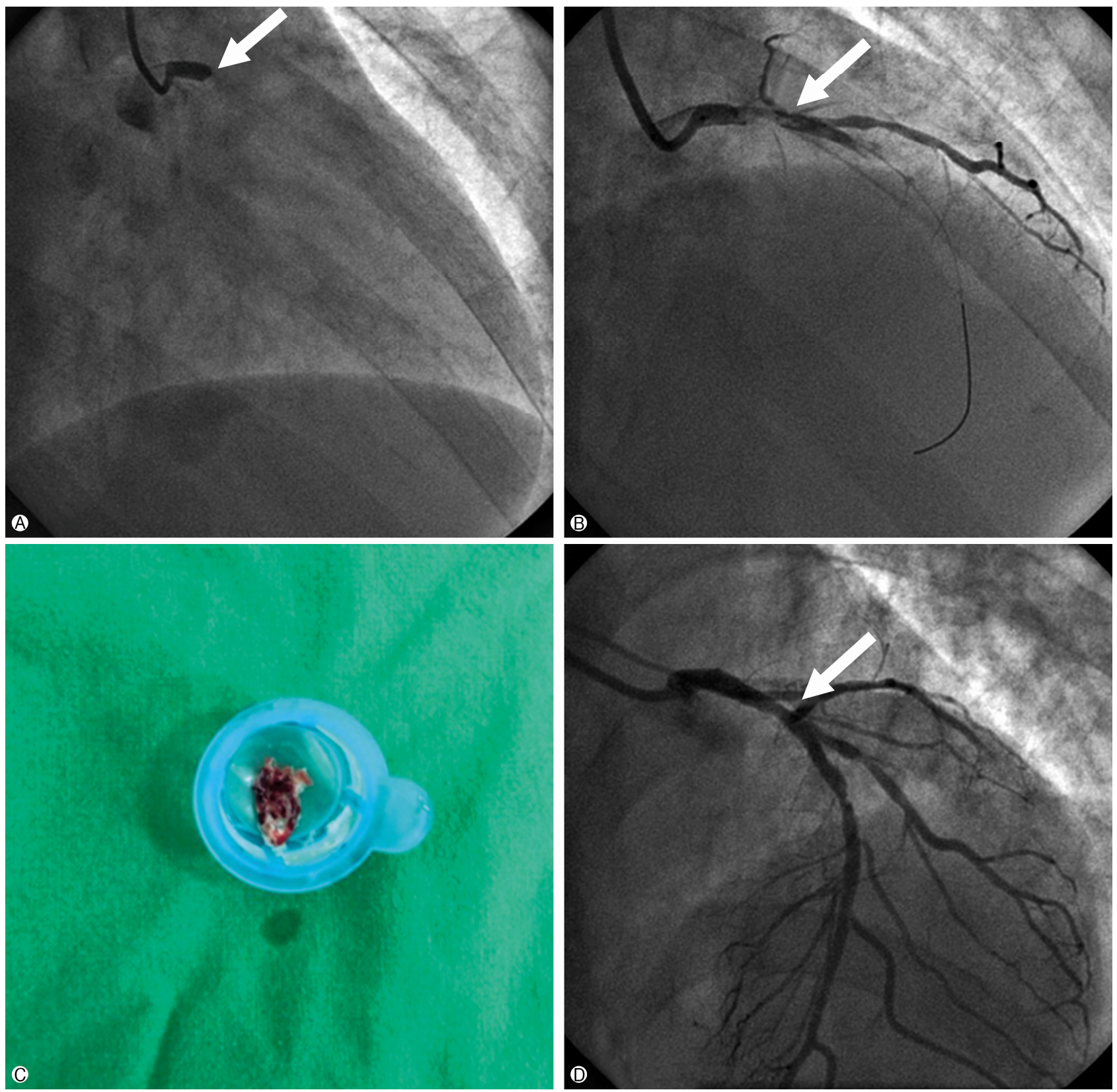

Fig. 2. Initial coronary angiographic findings. (A) Ttotal occlusion of left main coronary artery trunk. (B, C) Procedure of aspiration of thrombus and huge red thrombus was aspirated. (D) After thrombus aspiration, TIMI 3 flow was obtained.

was 12.7 per 10,000 person-years and women in the cohort were more likely to develop AMI than men (female hazard ratio $[\mathrm{HR}]=3.36$; male $\mathrm{HR}=2.01$ ) [7]. The reported risk of myocardial infarction in SLE patients ranges from 2- to 10fold greater than that in the general population, with a peak of 50-fold reported in women aged 35-44 years [8]. Our patient was a 40-year-old male and SLE was diagnosed after onset of AMI due to acute LMCA thrombosis. Hemolytic anemia helped lead to the correct diagnosis, and steroid therapy was immediately started.

A thrombotic event in SLE patients is mostly related to antiphospholipid syndrome (APS) [9]. APS manifests as venous or arterial thrombosis with abnormally elevated levels of serologic markers (anticardiolipin antibody and/or lupus anticoagulant). This patient did not have lupus anticoagulant or other serologic markers. 

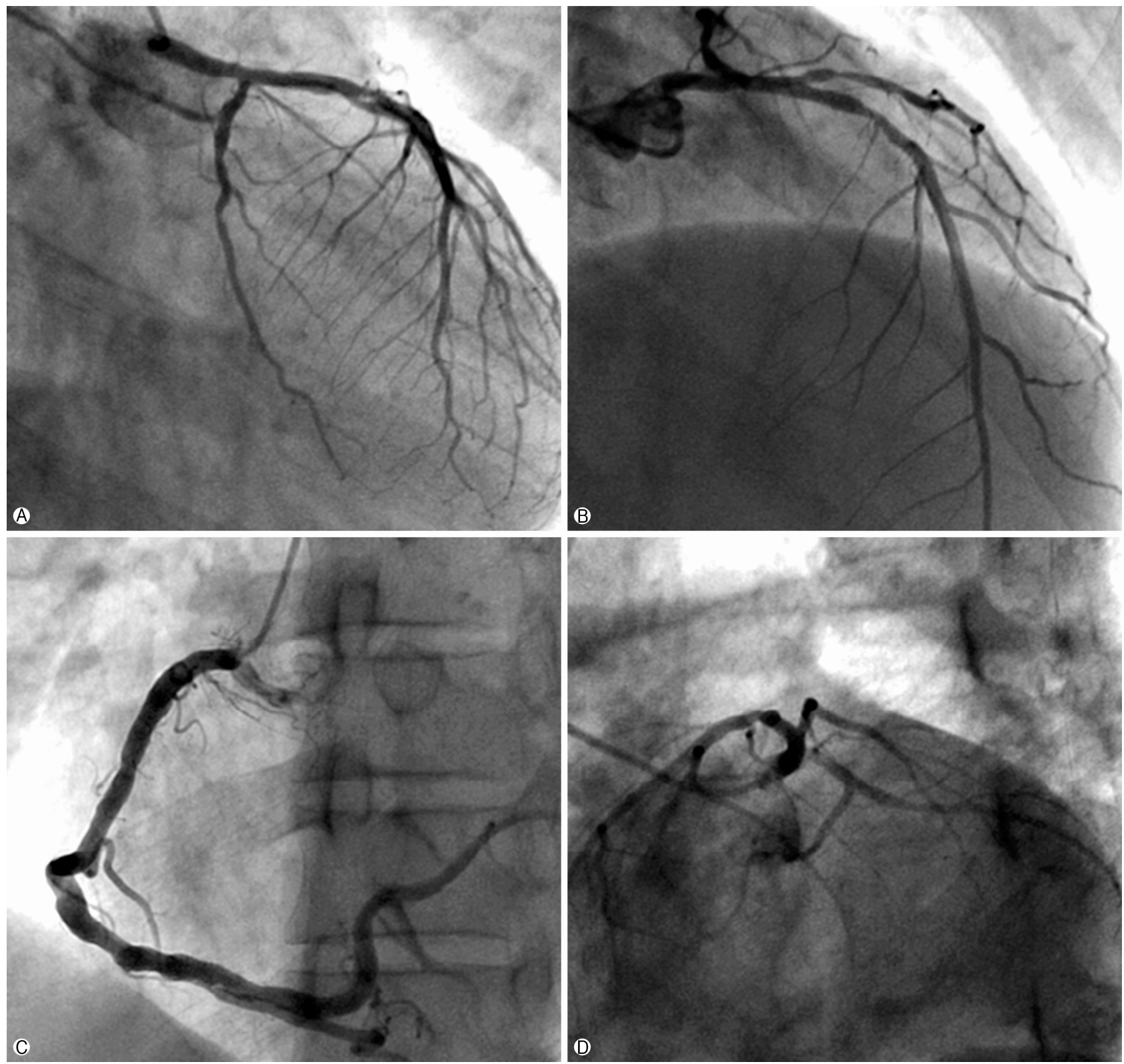

Fig. 3. Follow-up coronary angiographic findings at 1 year after index procedure (A-D). No luminal stenosis in left main coronary trunk $(\mathrm{C})$ and there is good patency in right coronary artery stent $(\mathrm{D})$.

There are no definitive guidelines regarding anticoagulation therapy in an SLE patient with thrombosis. In patients with APS and a thrombotic event, anticoagulation therapy is recommended [10]. In this case, only 1 month of anticoagulation with dual antiplatelet therapy for 1 year prevented further complications or clinical events. When the cause of coronary artery occlusion is a thrombus, aspiration may be helpful as a primary therapy. In many cases similar to ours, predilatation, thrombus aspiration, and stent implantation are routinely per- formed. Although stent implantation was not performed in this case, 1-year follow-up angiography showed good LMCA patency. This suggests that AMI in this patient was due to thrombosis, and evaluation of a lesion after thrombus aspiration can avoid unnecessary procedures, especially stent implantation.

In conclusion, SLE should be considered in the differential diagnosis in young patients who present with acute LMCA thrombosis. 


\section{CONFLICT OF INTEREST}

No potential conflict of interest relevant to this article was reported.

\section{ORCID}

Kang Un Choi, https://orcid.org/0000-0002-3385-3152

Ung Kim, https://orcid.org/0000-0002-6009-1843

\section{REFERENCES}

1. Klein AJ, Casserly IP, Messenger JC. Acute left main coronary arterial thrombosis - a case series. J Invasive Cardiol 2008;20: E243-6.

2. Jawaid A, Almas A. Cardiac tamponade as initial presentation in systemic lupus erythematosus. J Coll Physicians Surg Pak 2014;24(Suppl 2):S138-40.

3. Manautou L, Jerjes-Sanchez C, Meraz M, Perez-Garcia LF, Diaz-Cid A, de la Peña-Almaguer E, et al. Myopericarditis with predominantly right ventricular involvement with normal B-type natriuretic peptide and cardiac tamponade as the initial manifestation of systemic lupus erythematosus. Lupus 2014;23:935-8.

4. Kassaian SE, Goodarzynejad H, Darabian S, Basiri Z. Myocardial infarction secondary to premature coronary artery dis- ease as the initial major manifestation of systemic lupus erythematosus. Cardiovasc J Afr 2008;19:152-4.

5. Korkmaz C, Cansu DU, Kaşifoğlu T. Myocardial infarction in young patients ( $<$ or $=35$ years of age) with systemic lupus erythematosus: a case report and clinical analysis of the literature. Lupus 2007;16:289-97.

6. González-Pacheco H, Eid-Lidt G, Piña-Reyna Y, AmezcuaGuerra LM, Aldana-Sepúlveda N, Martínez-Sánchez C. Acute left main coronary artery thrombosis as the first manifestation of systemic lupus erythematosus and catastrophic antiphospholipid syndrome. Am J Emerg Med 2014;32:197.e3-5.

7. Chou CH, Lin CL, Chang SN, Lin MC, Kao CH, Huang YJ. A nationwide population-based retrospective cohort study: increased risk of acute myocardial infarction in systemic lupus erythematous patients. Int J Cardiol 2014;174:751-3.

8. Manzi S, Meilahn EN, Rairie JE, Conte CG, Medsger TA Jr, Jansen-McWilliams L, et al. Age-specific incidence rates of myocardial infarction and angina in women with systemic lupus erythematosus: comparison with the Framingham Study. Am J Epidemiol 1997;145:408-15.

9. Reynaud Q, Lega JC, Mismetti P, Chapelle C, Wahl D, Cathébras $\mathrm{P}$, et al. Risk of venous and arterial thrombosis according to type of antiphospholipid antibodies in adults without systemic lupus erythematosus: a systematic review and meta-analysis. Autoimmun Rev 2014;13:595-608.

10. Koniari I, Siminelakis SN, Baikoussis NG, Papadopoulos G, Goudevenos J, Apostolakis E. Antiphospholipid syndrome; its implication in cardiovascular diseases: a review. J Cardiothorac Surg 2010;5:101. 